\title{
Operating System Interface Becomes Non-Functional
}

National Cancer Institute

\section{Source}

National Cancer Institute. Operating System Interface Becomes Non-Functional. NCI

Thesaurus. Code C62894.

Problem associated with malfunction of the computer operating system as opposed to an application software problem. 